\title{
Erratum
}

\section{The influence of extracellular buffer concentration and propionate on lactate efflux from frog muscle}

\section{J. Mason, G. W. Mainwood, and J. S. Thoden}

Department of Physiology, University of Ottawa, 451 Smyth Road, Ottawa, Ontario K1H 8M5, Canada

Pflügers Arch (1986) 406:472- 479

Equations (5), (6) and (10) on page 476 of the above article should have read as follows:

$L H_{\mathrm{e}}=\frac{\left[L_{\mathrm{e}}^{-}\right]\left[H_{\mathrm{e}}^{+}\right]}{K_{L H}}$,

$L H_{\mathrm{e}}=\frac{\left[L_{\mathrm{e}}^{-}\right] \times\left[B H_{\mathrm{e}}\right]}{\left[B_{\mathrm{e}}^{-}\right]} \times \frac{K_{B}}{K_{L H}}$,

$L H_{\mathrm{c}}=\frac{J_{1}}{60 P_{L H} A}+L H_{\mathrm{i}}$

Also on page 476, in the right column, in the second paragraph of text between Eq. (9) and Eq. (10), $H_{e}$ should read $L H_{e}$. 